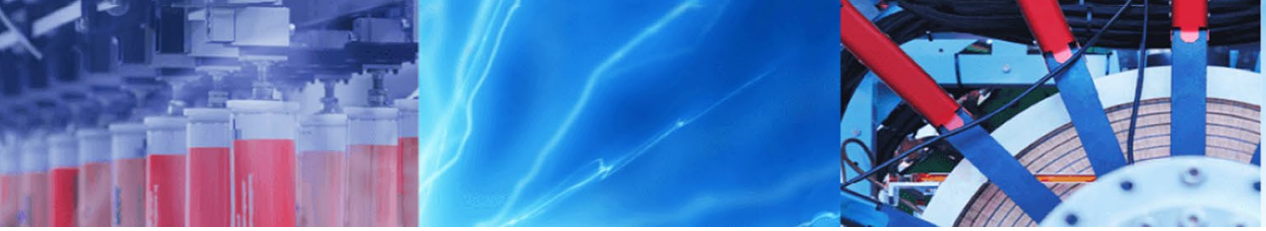

Research Article

\title{
Water hyacinth ash: an efficient green catalyst for the synthesis of $\beta$-amino carbonyl/nitrile compounds by aza-Michael reaction at room temperature
}

\author{
Anjumani Talukdar ${ }^{1}$. Dibakar Chandra Deka ${ }^{1,2}$
}

Received: 29 November 2019 / Accepted: 17 February 2020 / Published online: 10 March 2020

(c) Springer Nature Switzerland AG 2020

\begin{abstract}
Water hyacinth ash is found to be an efficient and reusable catalyst for the synthesis of $\beta$-amino carbonyl/nitrile compounds by aza-Michael reaction of amines with $a, \beta$-unsaturated compounds at room temperature under solvent free condition. Due to low cost of the catalyst, good activity, ease of handling and easy recovery with high yields of the products in short reaction time, easy workup procedure, mild reaction condition, the protocol is highly applicable in synthetic organic chemistry.
\end{abstract}

Keywords Aza-Michael $\cdot$ Water hyacinth $\cdot \beta$-amino carbonyl $\cdot \beta$-amino nitrile $\cdot$ Solvent free

\section{Introduction}

Development of simple, green and cost effective synthetic procedure is the main focus of the modern synthetic chemist. In this context, biomass-derived heterogeneous catalysts play a key role due to wide range of advantages such as low cost, high activity, easy recovery and reusability. Recently, the water extracts of various agro-waste ashes have been successfully employed as green and nonconventional solvent or as green catalysts in a number of organic reactions viz. Suzuki-Miyaura [1-7] and Sonogashira cross-coupling [8, 9], Dakin reaction [10], ipso-hydroxylation of arylboronic acid [11], Knoevenagel reaction [12], Henry reaction [13], 3-carboxycoumarins synthesis [14], peptide bond synthesis [15], etc. Additionally, many researchers have reported the ash based heterogeneous catalysts derived from different agricultural wastes as the promising green catalysts for biodiesel production. Some of the reported heterogeneous catalysts from agro-wastes for biodiesel productions are Musa balbisiana [16-18], Lemna perpusilla [19], coconut husk [20], rice husk [21], cocoa pod husk [22], Carica papaya stem [23], Tectona grandis leaves [24], etc.

Water hyacinth is an aquatic weed which is naturally available across the globe in fresh water wet lands. Water hyacinth ash (WHA) has been found an efficient basic heterogeneous catalyst for organic transformations $[25,26]$. Its high stability, ease of handling, reusability, non-toxicity and eco-friendliness make it a catalyst of choice.

The $\beta$-amino carbonyl/nitrile compounds have found a wide range of applications for the synthesis of a variety of bioactive natural products [27], antibiotics [28], heterocyclic compounds [29], chiral auxiliaries [30] etc. Further, its presence as structural constituents in biologically active natural products and use in the synthesis of chemicals [31] and pharmaceuticals [32] have elevated its

Electronic supplementary material The online version of this article (https://doi.org/10.1007/s42452-020-2281-7) contains supplementary material, which is available to authorized users.

Dibakar Chandra Deka, dcdeka@rediffmail.com | 'Department of Chemistry, Gauhati University, Guwahati, Assam 781014, India. ${ }^{2}$ Present Address: Madhabdev University, PO Dikrong, Narayanpur, Assam 784164, India. 
synthetic importance. Among the various methods, the aza-Michael addition is a straightforward route for the generation of carbon-nitrogen bonds in the synthesis of $\beta$-amino carbonyl or nitrile compounds. Aza-Michael reaction is reportedly catalyzed by a couple of transition metal salts like $\mathrm{InCl}_{3}$ [33], $\mathrm{CeCl}_{3} \cdot 7 \mathrm{H}_{2} \mathrm{O}$ [34], $\mathrm{Yb}(\mathrm{OTf})_{3}$ [35], $\mathrm{Bi}\left(\mathrm{NO}_{3}\right)_{3}$ [36], $\mathrm{Cu}(\mathrm{OTf})_{2}$ [37], $\mathrm{LiClO}_{4}$ [38], $\mathrm{Sml}_{2}$ [39], $\mathrm{FeCl}_{3} \cdot 6 \mathrm{H}_{2} \mathrm{O}$ [40], cerium(IV) ammonium nitrate [41]. Heterogeneous catalysts like $\mathrm{SiO}_{2}-\mathrm{H}_{2} \mathrm{SO}_{4}[42], \mathrm{SiO}_{2}-\mathrm{HClO}_{4}[43]$ have also been found to effectively catalyze the reaction. However, most of the protocols are associated with one or more limitations such as harsh reaction condition, high temperature, use of expensive catalyst, toxic organic solvent and tedious workup procedure. Therefore, the development of a simple, efficient and cost-effective methodology under mild reaction condition is highly desirable. In continuation of our research in the application of bio-mass derived heterogeneous catalysts in organic synthesis, herein we are reporting a simple and environmentally benign catalyst derived from the waste biomass of water hyacinth to catalyze aza-Michael addition of amines (1) to $\alpha, \beta$-unsaturated compounds (2) at room temperature under solvent free condition (Scheme 1).

\section{Experimental}

All commercially available chemicals and reagents were purchased from Aldrich and used without further purification. IR spectra were recorded on a SHIMADZU FT-IR instrument as $\mathrm{KBr}$ pellets and the adsorption expressed in $\mathrm{cm}^{-1}$. ${ }^{1} \mathrm{H}(300 \mathrm{MHz})$ and ${ }^{13} \mathrm{C}-\mathrm{NMR}(75 \mathrm{MHz})$ spectra were recorded on a Bruker NMR spectrometer at ambient temperature. Chemical shifts are given in $\delta$ relative to tetramethylsilane (TMS), the coupling constants $J$ are given in $\mathrm{Hz}$. The spectra were recorded in $\mathrm{CDCl}_{3}$ or DMSO- $\mathrm{d}_{6}$ as solvent at ambient temperature, TMS served as internal standard $(\delta=0 \mathrm{ppm})$ for ${ }^{1} \mathrm{H}$ NMR. Mass spectra were recorded on Waters ZQ 4000 equipped with ESI source. Elemental analysis was done on Perkin Elmer Series II Analyszer 2400. Column chromatography was performed on silica gel (60-120 mesh) using ethyl acetate:petroleum ether as the eluent. For TLC, precoated silica gel plates (Merck 60 F254) were used and compounds were visualized by irradiating with UV light or in an iodine chamber.

\subsection{General procedure for the synthesis of $\beta$-amino carbonyl compounds}

A mixture of aliphatic amine ( $3 \mathrm{mmol}), \alpha, \beta$-unsaturated compound $(3.3 \mathrm{mmol})$, and $10 \mathrm{mg}$ of the WH ash was stirred at ambient temperature for a certain period of time. The progress of the reaction was monitored by TLC. After completion of the reaction, the catalyst was filtered off and the mixture was extracted with ethyl acetate thrice ( 25 $m L x 3)$. The combined extract was dried over anhydrous sodium sulfate and the solvent was removed in a rotary evaporator under reduced pressure. The crude product was purified by column chromatography on silica-gel with different ratios of petroleum ether/ethyl acetate as the eluent. The recovered catalyst was washed repeatedly with ethyl acetate and acetone, dried in hot air oven and reused for subsequent experiments.

\subsection{Preparation of the catalyst}

Roots were separated from the plant stems and were cut into small pieces and dried under sun for about 15 days. The dry mass was burnt in an earthen pot in the presence of air. The ash was allowed to cool down to ambient temperature in its own and preserved in an air tight container. The water hyacinth ash (WHA) was further heated in a furnace at $500^{\circ} \mathrm{C}$ for $3 \mathrm{~h}$ and kept in a desiccator before use. From proximate analyses it was found that the ash contains $48.87 \mathrm{wt} \%$ moisture at $110^{\circ} \mathrm{C}$ and $7 \mathrm{wt} \%$ volatile matters at $350^{\circ} \mathrm{C}$ [26].

\subsubsection{Catalyst composition}

The chemical composition of the catalyst was examined by chemical analysis and different standard laboratory techniques such as SEM-EDX, XRF, TEM, atomic absorption spectroscopy and flame photometry. Potassium and
Scheme 1 Aza-Michael addition of amines to $\alpha, \beta$ unsaturated carbonyl/nitrile compounds

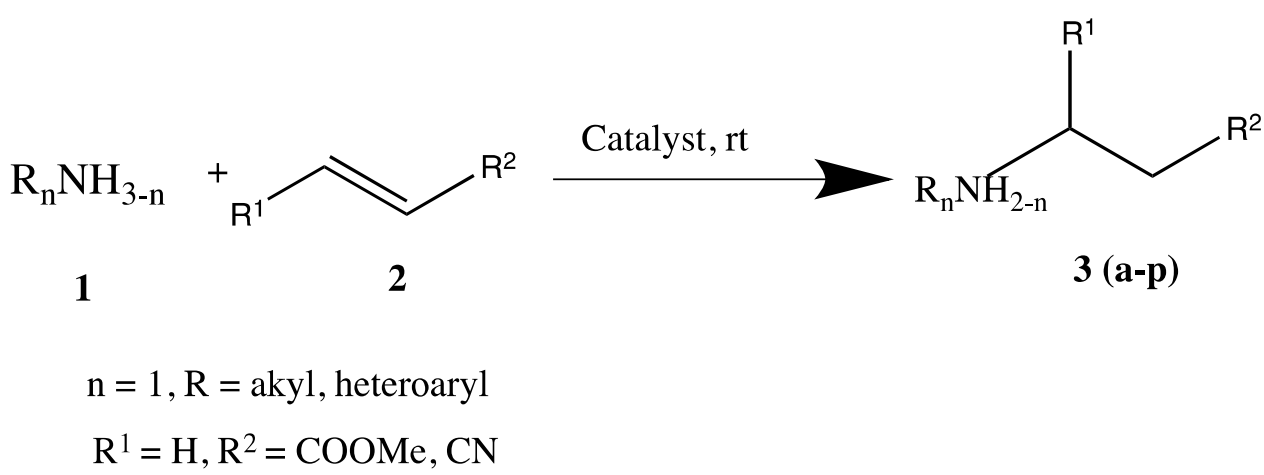


calcium are present as the major components along with eleven other metals viz. $\mathrm{Na}, \mathrm{Fe}, \mathrm{Mg} \mathrm{Cr}, \mathrm{Mn}, \mathrm{Co}, \mathrm{Ni}, \mathrm{Cu}, \mathrm{Zn}, \mathrm{Pb}$ and Ti which are present only in trace amounts (ppm level). Metals are present in the form of carbonates, chlorides, sulfates, phosphates and oxides $[26,44]$.

\section{Results and discussion}

In significance, this methodology proves to be operationally simple affording high yields in short reaction time. Further, achieving the synthesis under solvent free condition and at room temperature makes it cost and energy efficient. The application of water hyacinth ash (WHA) as a heterogeneous catalyst further expands wider scope of the less explored biomass derived natural catalysts in organic synthesis. Moreover, simple purification process along with reusablity of the catalyst up to three times increases the applicability of the methodology.

Initially, methyl acrylate and benzylamine were chosen to optimize the catalyst loading at ambient temperature (Table 1) to generate the corresponding Michael adduct as shown in Scheme 1.The reaction was carried out in the presence of $0.01 \mathrm{~g}$ of ash in solvent free condition at ambient temperature. After 20 min complete disappearance of starting material was observed (monitored by TLC). On increasing the catalyst loading from 0.01 to $0.02 \mathrm{~g}$, reaction time was decreased to $10 \mathrm{~min}$ with $95 \%$ yield. The reaction was attempted in different solvents such as dichloromethane, methanol and ethanol, but the yield was found to be better in absence of the solvents. Under the optimized conditions, we then explored the scope of the reaction. As shown in Table 3 , a wide range of $\beta$-amino carbonyl and nitrile compounds were prepared from the reactions of different primary and secondary amines (both acyclic and cyclic), and various $\alpha, \beta$-unsaturated compounds and Baylis-Hillman adducts. When amine and alkene ratio was $1: 2$, doubly substituted products were isolated (Table 3 , entry 10).

Having optimized the reaction condition we then proceeded to examine the efficiency of the catalyst. The efficiency of WHA as a catalyst was compared to common bases like $\mathrm{K}_{2} \mathrm{CO}_{3}, \mathrm{Na}_{2} \mathrm{CO}_{3}$ and Lewis acids such as $\mathrm{FeCl}_{3} \cdot 6 \mathrm{H}_{2} \mathrm{O}$ and $\mathrm{SnCl}_{4}$ with results as shown in Table 2 . WHA shows better catalytic activity as compared to $\mathrm{K}_{2} \mathrm{CO}_{3}$, $\mathrm{Na}_{2} \mathrm{CO}_{3}, \mathrm{FeCl}_{3}$ and $\mathrm{SnCl}_{4}$.

Under optimized reaction conditions, all the amines afforded the desired product in good yield (Table 3). After completion of the reaction, the catalyst could be easily recovered from the reaction mixture and reused in several runs.

The reusability of the catalyst was studied through a model reaction of 2-aminoethanol and methyl acrylate under the optimized conditions, where the catalyst was separated by simple filtration, washed with ethyl acetate and acetone for several times and dried in a hot air oven and reused. It was observed that WHA was reusable but with a bit of sacrifice in yield in every subsequent run (Fig. 1).

\section{Conclusion}

In conclusion, water hyacinth ash is an efficient catalyst for Michael addition of amines with different Michael acceptors including Baylis-Hilman products under solventfree condition. The procedure avoids the use of organic solvent to carry out the reaction, and the catalyst can be separated from the product quite easily and reused. The catalyst is inexpensive, environment-friendly and can be disposed off without affecting the environment.

\section{Spectral data}

Compound 3a (Entry 1, Table 3): 2-[(Ethylamino)methyl]-3-hy-<smiles>CCNCC(C#N)C(O)c1ccccc1</smiles>

droxy-3-phenylpropanenitrile, $\mathrm{C}_{12} \mathrm{H}_{16} \mathrm{~N}_{2} \mathrm{O} 0.188$ g; yield 92\%; colourless solid; m.p. $68^{\circ} \mathrm{C}$; ${ }^{1} \mathrm{H}$ NMR $\left(300 \mathrm{MHz}, \mathrm{CDCl}_{3}\right)$ $\delta_{\mathrm{H}}: 1.12(\mathrm{t}, 3 \mathrm{H}), 2.68(\mathrm{q}, 2 \mathrm{H}), 2.74(\mathrm{~m}, 2 \mathrm{H}), 3.77(\mathrm{~m}, 2 \mathrm{H})$, 5.02(1H), $7.38(\mathrm{~m}, 5 \mathrm{H}) ;{ }^{13} \mathrm{C} \mathrm{NMR}\left(75 \mathrm{MHz}, \mathrm{CDCl}_{3}\right) \delta_{\mathrm{c}}: 140.64$, $128.45,125.73,118.41,75.13,39.98,38.36,14.77 ; \mathrm{MS}$ (ESI) $\mathrm{m} / \mathrm{z}$ 204.27($\left(\mathrm{M}^{+}\right)$; Elemental analysis: calculated: $\mathrm{C}, 70.56, \mathrm{H}$ 7.90, N, 13.71; found: C, 70.63, H 7.82, N, 13.61.

Compound 3b (Entry 2, Table 3): 2-[(Dimethylamino)methyl]-3-hydroxy-3-phenylpropanenitrile,

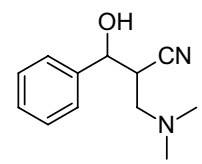

$\mathrm{C}_{12} \mathrm{H}_{16} \mathrm{~N}_{2} \mathrm{O} 0.192 \mathrm{~g}$; yield $94 \%$; colourless solid; $46{ }^{\circ} \mathrm{C} ;{ }^{1} \mathrm{H}$ $\operatorname{NMR}\left(300 \mathrm{MHz}, \mathrm{CDCl}_{3}\right) \delta_{\mathrm{H}}: 2.37,2.72(\mathrm{~d}, 1 \mathrm{H}), 2.79(\mathrm{~d}, 1 \mathrm{H})$, 3.08 (broad peak, $1 \mathrm{H}-\mathrm{OH}), 5.08(\mathrm{~s}, 1 \mathrm{H}), 7.33-7.46(\mathrm{~m}, 5 \mathrm{H}$, $\mathrm{Ar} \mathrm{H}) ;{ }^{13} \mathrm{C}$ NMR $\left(75 \mathrm{MHz} \mathrm{CDCl}_{3}\right) \delta_{\mathrm{C}}: 139.95,128.44,128.39$, $128.15,128.36,125.94,118.59,73.72,58.69,45.83,37.08 \mathrm{MS}$ (ESI) $\mathrm{m} / \mathrm{z}$ 204.27( $\left(\mathrm{M}^{+}\right)$; Elemental analysis: calculated: $\mathrm{C}$ 70.56, H, 7.90, N, 13.71; found: C, 70.46, H, 7.80, N, 13.66 .

Compound 3c (Entry 3, Table 3): 2-[(Dimethylamino)methyl]-3-hydroxy-3-(p-tolyl)propanenitrile, 
<smiles>Cc1ccc(C(O)C(C#N)CN(C)C)cc1</smiles>

$\mathrm{C}_{13} \mathrm{H}_{18} \mathrm{~N}_{2} \mathrm{O} 0.198 \mathrm{~g}$; yield $91 \%$; Colourless solid; $38^{\circ} \mathrm{C}^{\circ}{ }^{1} \mathrm{H}$ $\operatorname{NMR}\left(300 \mathrm{MHz}, \mathrm{CDCl}_{3}\right) \delta_{\mathrm{H}}: 2.36(\mathrm{~s}, 9 \mathrm{H}), 2.68(\mathrm{~d}, 1 \mathrm{H}), 2.74(\mathrm{~d}$, 1H), 3.06 (broad peak, $-\mathrm{OH}), 5.03(\mathrm{~s}, 1 \mathrm{H}), 7.18(\mathrm{~d}, 2 \mathrm{H} \mathrm{Ar}$ ), 7.31 (s, $2 \mathrm{H} \mathrm{Ar}) ;{ }^{13} \mathrm{C}$ NMR $(75 \mathrm{MHz}, \mathrm{CDCl} 3) \delta_{\mathrm{C}}: 137.89,137.02$, $129.14,129.09,126.28,125.87,118.78,73.54,58.64,45.82$, 45.33, 37.23, 36.70, 21.08; MS (ESI) m/z 218.14( $\left.\mathrm{M}^{+}\right)$; Elemental analysis: calculated: $\mathrm{C}, 71.53, \mathrm{H}, 8.31, \mathrm{~N}, 12.83$; found: $\mathrm{C}$, $71.41, \mathrm{H}, 8.20, \mathrm{~N}, 12.71$.

Table 1 Optimization of reaction conditions $\mathrm{s}^{\mathrm{a}}$
Compound 3d (Entry 4, Table 3): 2-((Ethylamino)methyl)-3-hydroxy-3-(pyridin-3-yl)propanenitrile,<smiles>CCNCC(C#N)C(O)c1cccnc1</smiles>

$\mathrm{C}_{11} \mathrm{H}_{15} \mathrm{~N}_{3} \mathrm{O} 0.168$ g; yield $82 \%$; dark brown oily compound; ${ }^{1} \mathrm{H} \mathrm{NMR}\left(300 \mathrm{MHz}, \mathrm{CDCl}_{3}\right) \delta_{\mathrm{H}}: 1.06(\mathrm{q}, 3 \mathrm{H}), 1.87(\mathrm{~s}, 1 \mathrm{H}), 2.65(\mathrm{~m}$, $3 \mathrm{H}), 3.05(\mathrm{~m}, 1 \mathrm{H}), 3.36(\mathrm{~m}, 2 \mathrm{H}), 5.04(\mathrm{~s}, 1 \mathrm{H}), 7.19(\mathrm{~d}, 1 \mathrm{H}), 7.54$ $(\mathrm{d}, 1 \mathrm{H}), 7.72(\mathrm{~d}, 1 \mathrm{H}), 8.48(\mathrm{dd}, 1 \mathrm{H}){ }^{13} \mathrm{C} \mathrm{NMR}\left(75 \mathrm{MHz}, \mathrm{CDCl}_{3}\right)$ $\delta_{C}: 159.38,148.69,137.40,121.82,74.21,44.17,39.06,15.12$;

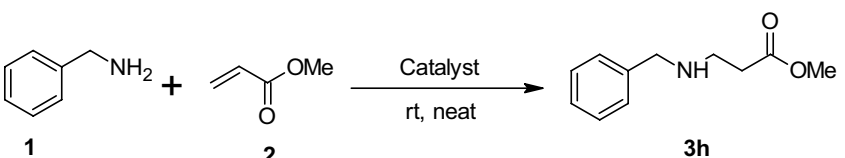

\begin{tabular}{lllll}
\hline Entry & Catalyst $(\mathrm{g})$ & Solvent & Time $(\mathrm{min})$ & Yield $(\%)^{\mathrm{b}}$ \\
\hline 1 & - & - & 180 & 180 \\
2 & Ethanol & 180 & 30 \\
3 & 0.002 & Ethanol & 120 & 50 \\
4 & 0.004 & Ethanol & 90 & 80 \\
5 & 0.006 & Ethanol & 20 & 91 \\
6 & 0.008 & Ethanol & 30 & 94 \\
7 & 0.01 & Methanol & 60 & 45 \\
8 & 0.01 & Dichloromethane & 20 & 95 \\
9 & 0.01 & No solvent & 10 & 95 \\
10 & 0.01 & No solvent & & \\
\hline
\end{tabular}

${ }^{\mathrm{a}}$ Reaction conditions: benzylamine $(1 \mathrm{mmol})$, methyl acrylate $(1 \mathrm{mmol}),{ }^{\mathrm{b}}$ Isolated yields

Table 2 Effect of various catalysts on the model reaction of benzylamine and methyl acrylate ${ }^{a}$

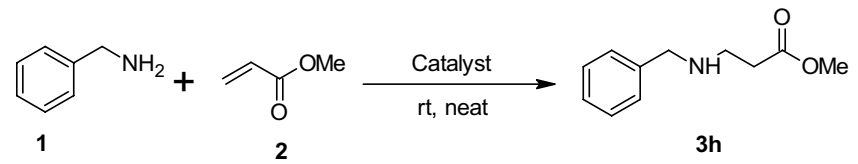

\begin{tabular}{lll}
\hline Entry & Catalyst & Yield $^{\mathrm{b}}(\%)$ \\
\hline 1 & $\mathrm{~K}_{2} \mathrm{CO}_{3}$ & 60 \\
2 & $\mathrm{Na}_{2} \mathrm{CO}_{3}$ & 50 \\
3 & $\mathrm{FeCl}_{3}$ & 58 \\
4 & $\mathrm{SnCl}_{4}$ & 87 \\
5 & $\mathrm{WHA}$ & 96 \\
\hline
\end{tabular}

a Reaction conditions: benzyl amine (1 mmol), methyl acrylate $(1 \mathrm{mmol})$, WHA $(10 \mathrm{mg})$, ${ }^{\text {bl }}$ solated yields 
Table 3 Aza-Michael addition of amines to $\alpha, \beta$-unsaturated compounds (Scheme 1$)^{\text {a }}$

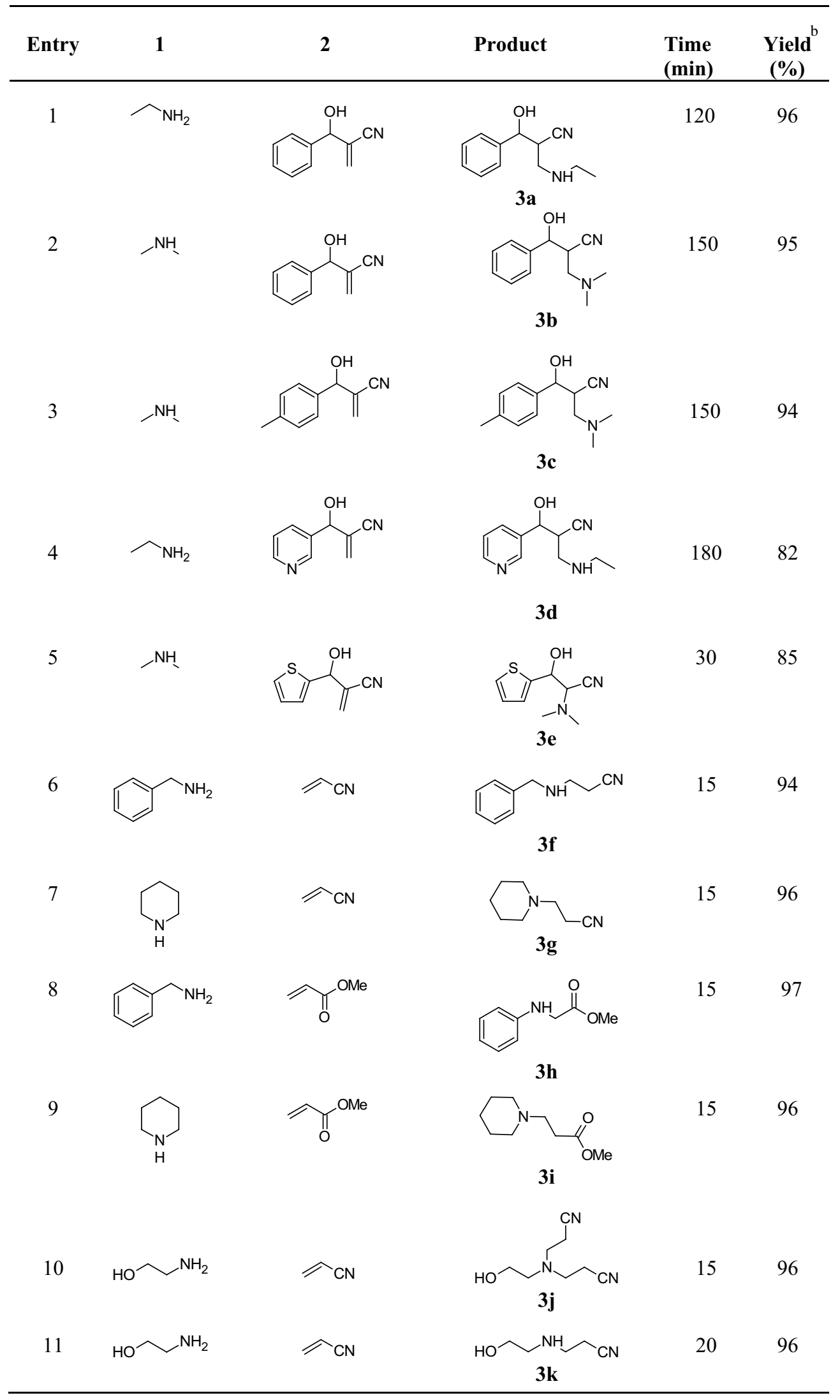


Table 3 (continued)<smiles>C=CC(=O)OCC(=O)C(O)c1ccccc1C(=O)C(=C)C(=O)OC</smiles>

${ }^{a}$ The products are characterized by their spectral data (IR, ${ }^{1} \mathrm{H}$ NMR, ${ }^{13} \mathrm{C}$ NMR, Mass spectra); ${ }^{\mathrm{b}}$ Isolated yield.

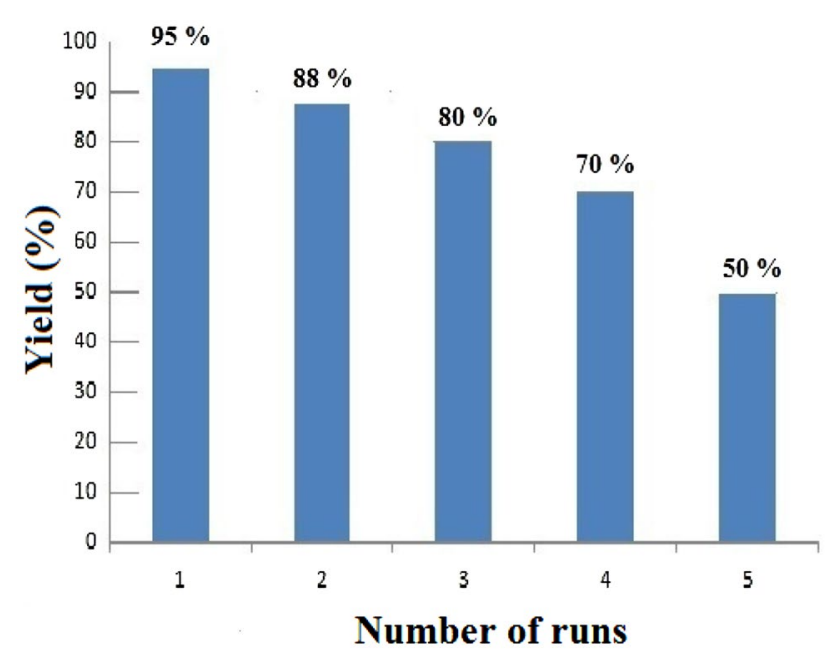

Fig. 1 Recyclability of WHA

MS (ESI) m/z 205.26( $\left.\mathrm{M}^{+}\right)$; Elemental analysis: calculated: $\mathrm{C}$, 64.37, H 7.37, N, 20.47; found: C, 64.26, H 7.24, N, 20.40.

Compound 3e (Entry 5, Table 3): 2-[(Ethylamino) methyl]-3-hydroxy-3-(thiophen-2-yl)propanenitrile,<smiles>CN(C)C(C#N)C(O)c1cccs1</smiles>

$\mathrm{C}_{10} \mathrm{H}_{14} \mathrm{~N}_{2}$ OS 0.179 g; yield $85 \%$; black oily compound; ${ }^{1} \mathrm{H}$ NMR (300 MHz, CDCl $)_{3} \delta_{\mathrm{H}}: 2.26(\mathrm{~m}, 6 \mathrm{H}), 2.45(\mathrm{~m}, 2 \mathrm{H}), 4.28$ $(\mathrm{m}, 2 \mathrm{H}), 6.94-7.46(\mathrm{~m}, 4 \mathrm{H}) ;{ }^{13} \mathrm{C} \mathrm{NMR}\left(75 \mathrm{MHz} \mathrm{CDCl}_{3}\right) \delta_{\mathrm{C}}$ : 138.16, 127.36, 125.15, 124.08, 62.55, 45.05, 37.37; MS (ESI) $\mathrm{m} / \mathrm{z} 210.30\left(\mathrm{M}^{+}\right)$; Elemental analysis: calculated: $\mathrm{C}, 57.11, \mathrm{H}$, 6.71, N13.32; found: C, 57.08, H, 6.66, N, 13.21.

Compound 3f (Entry 6, Table 3): 3-(Benzylamino)propaneni-<smiles>N#CCCNCc1ccccc1</smiles>

trile, $\mathrm{C}_{10} \mathrm{H}_{12} \mathrm{~N}_{2}$ Yield 95\%; white solid; $\mathrm{mp} 46{ }^{\circ} \mathrm{C} ;{ }^{1} \mathrm{H}$ NMR $\left(\mathrm{CDCl}_{3}, 300 \mathrm{MHz}\right) \delta_{\mathrm{H}}: 7.34(\mathrm{~m}, 5 \mathrm{H}), 3.85(\mathrm{~s}, 2 \mathrm{H}), 2.97(\mathrm{t}$, $J=6.3 \mathrm{~Hz}, 2 \mathrm{H}), 2.58(\mathrm{t}, J=6.27 \mathrm{~Hz}, 2 \mathrm{H}), 1.69(\mathrm{br} \mathrm{s}, 1 \mathrm{H}) ;{ }^{13} \mathrm{C}$ NMR $\left(\mathrm{CDCl}_{3}, 75 \mathrm{MHz}\right) \delta_{\mathrm{C}}: 139.35,128.59,128.09,127.33$, 118.74, 53.18, 44.30, 18.78; MS (ESI, m/z) $161.8\left(\mathrm{M}^{+}+1\right)$. Elemental analysis: calculated: $\mathrm{C}, 74.97, \mathrm{H}, 7.55, \mathrm{~N}, 17.48$; found: $\mathrm{C}, 75.23, \mathrm{H}, 7.84, \mathrm{~N}, 16.10$. 
Compound 3 g (Entry 7, Table 3): 3-(Piperidin-1-yl)propanen-

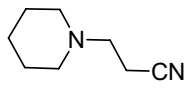

itrile, $\mathrm{C}_{8} \mathrm{H}_{14} \mathrm{~N}_{2} 0.133$ g; yield $96 \%$; yellow oily compound; ${ }^{1} \mathrm{H}$ NMR $\left(300 \mathrm{MHz}, \mathrm{CDCl}_{3}\right) \delta_{\mathrm{H}}: 1.43(\mathrm{~m}, 2 \mathrm{H}), 1.55(\mathrm{~m}, 4 \mathrm{H})$, $2.39(\mathrm{~m}, 4 \mathrm{H}), 2.45(\mathrm{~m}, 2 \mathrm{H}), 2.64(\mathrm{~m}, 2 \mathrm{H}) ;{ }^{13} \mathrm{C}$ NMR $(75 \mathrm{MHz}$, $\mathrm{CDCl} 3) \delta_{\mathrm{C}}: 118.96,53.96,53.81,25.61,23.89,15.43 . \mathrm{MS}$ (ESI) $\mathrm{m} / \mathrm{z} 139.1\left(\mathrm{M}^{+}+1\right)$; Elemental analysis: calculated: $C, 69.52$, $\mathrm{H}, 10.21, \mathrm{~N}, 20.27$; found: $\mathrm{C}, 57.46, \mathrm{H}, 7.84, \mathrm{~N}, 25.13$.

\section{Compound $3 \mathrm{~h}$ (Entry 8, Table 3): Methyl 3-(benzylamino)pro-}<smiles>COC(=O)CNc1ccccc1</smiles>

panoate, $\mathrm{C}_{11} \mathrm{H}_{15} \mathrm{NO}_{2} 0.175 \mathrm{~g}$ (90\%); colourless oily liquid; ${ }^{1} \mathrm{H}$ $\operatorname{NMR}\left(300 \mathrm{MHz}, \mathrm{CDCl}_{3}\right): \delta_{\mathrm{H}}: 2.45(\mathrm{t}, 2 \mathrm{H}), 2.47(\mathrm{~s},-\mathrm{NH}), 2.80(\mathrm{q}$, $2 \mathrm{H}), 3.58(\mathrm{~s}, 3 \mathrm{H}), 3.71(\mathrm{~s}, 2 \mathrm{H}), 7.21(\mathrm{~m}, \mathrm{Ar} \mathrm{H}) ;{ }^{13} \mathrm{C} \mathrm{NMR}(75 \mathrm{MHz}$, DMSO) $\delta_{C}: 172.65,139.33,127.96,127.70,126.57,53.11$, 51.10, 43.83, 33.83, 33.83; MS(ESI) m/z: $194.3\left(M^{+}+1\right)$; Elemental analysis: calculated: $\mathrm{C}, 68.37, \mathrm{H}, 7.82, \mathrm{~N}, 7.25$; found: C, 68.32, H, 7.69, N, 7.20.

Compound 3i (Entry 9, Table 3): Methyl 3-(piperidin-1-yl)pro-

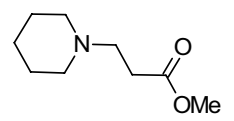

panoate, $\mathrm{C}_{9} \mathrm{H}_{17} \mathrm{NO}_{2} 0.159 \mathrm{~g}(90 \%)$, yellow liquid; ${ }^{1} \mathrm{H}$ NMR $\left(300 \mathrm{MHz}, \mathrm{CDCl}_{3}\right): \delta_{\mathrm{H}}: 1.06(\mathrm{~s}, 1 \mathrm{H}), 1.32(\mathrm{~d}, 2 \mathrm{H}), 1.43(\mathrm{~d}, 4 \mathrm{H})$, $2.28(\mathrm{~s}, 3 \mathrm{H}), 2.39(\mathrm{~m}, 2 \mathrm{H}), 2.49(\mathrm{~m}, 2 \mathrm{H}), 3.55(\mathrm{~s}, 3 \mathrm{H}){ }^{13} \mathrm{C} \mathrm{NMR}$ $\left(75 \mathrm{MHz}, \mathrm{CDC}_{13}\right) \delta_{\mathrm{C}}: 172.18,54.03,40.06,31.74,25.71,24.36$; MS (ESI) m/z $172.3\left(M^{+}+1\right)$; Elemental analysis: calculated: C, 63.13, H, 10.01, N, 8.18; found: $C, 63.08, H, 10.00, N, 8.10$.

Compound 3j (Entry 10, Table 3): 3,3-[(2-Hydroxyethyl)<smiles>N#CCCN(CCO)CCO</smiles>

azanediyl]dipropanenitrile, $\mathrm{C}_{8} \mathrm{H}_{13} \mathrm{~N}_{3} \mathrm{O} 0.151 \mathrm{~g}(90 \%)$, yellow oily compound; ${ }^{1} \mathrm{H}$ NMR $(300 \mathrm{MHz}, \mathrm{CDCl} 3): \delta_{\mathrm{H}}: 2.45$ $(\mathrm{t}, 2 \mathrm{H}), 2.59(\mathrm{t}, 2 \mathrm{H}), 2.75(\mathrm{t}, 2 \mathrm{H}), 3.46(\mathrm{q}, 2 \mathrm{H}), 3.67(\mathrm{~m}, 5 \mathrm{H})$; ${ }^{13} \mathrm{C}$ NMR $(75 \mathrm{MHz}, \mathrm{CDCl} 3) \delta_{\mathrm{C}}: 120.91,76.81,58.82,49.28$, 42.47, 16.61. MS (ESI) $\mathrm{m} / \mathrm{z} 168.2\left(\mathrm{M}^{+}+1\right)$; Elemental analysis: calculated: $\mathrm{C}, 57.46, \mathrm{H}, 7.84, \mathrm{~N}, 25.13$; found: $\mathrm{C}, 57.32$, $\mathrm{H}, 7.70, \mathrm{~N}, 25.09$.
Compound 3 k (Entry 11, Table 3): 3-(2-hydroxyethylamino)

$$
\text { HO } \sim \mathrm{NH} \frown \mathrm{CN}
$$

propanenitrile $0.151 \mathrm{~g}(90 \%)$, brown oily compound; ${ }^{1} \mathrm{H}$ NMR $(300 \mathrm{MHz}, \mathrm{CDCl} 3) \delta_{\mathrm{H}}: 2.13(\mathrm{~s}, 2 \mathrm{H}), 2.48(\mathrm{t}, 2 \mathrm{H}), 2.74$ $(\mathrm{t}, 2 \mathrm{H}), 2.87(\mathrm{t}, 2 \mathrm{H}), 3.6(\mathrm{t}, 2 \mathrm{H}) ;{ }^{13} \mathrm{C}$ NMR $(75 \mathrm{MHz}, \mathrm{CDCl} 3)$ $\delta_{C}: 118.74,60.41,52.03,44.28,23.29$. MS (ESI) $\mathrm{m} / \mathrm{z} 168.2$ $\left(\mathrm{M}^{+}+1\right)$; Elemental analysis: calculated: $\mathrm{C}, 57.46, \mathrm{H}, 7.84, \mathrm{~N}$, 25.13; found: C, 57.32, H, 7.70, N, 25.09.

Compound 3 I (Entry 12, Table 3): Methyl 4-((2-hydroxy-<smiles>COC(=O)CCNCO</smiles>

ethyl)amino)butanoate, $\mathrm{C}_{7} \mathrm{H}_{15} \mathrm{NO}_{3} 0.146 \mathrm{~g}(90 \%)$; brown oily compound; ${ }^{1} \mathrm{H}$ NMR (300 MHz, DMSO) $\delta_{H}: 2.54(\mathrm{~m}$, 7H), $2.71(\mathrm{~m}, 2 \mathrm{H}), 3.42(\mathrm{t}, 1 \mathrm{H}), 3.57(\mathrm{~m}, 3 \mathrm{H}) ;{ }^{13} \mathrm{C} \mathrm{NMR}(75 \mathrm{MHz}$, DMSO) $\delta_{C}: \delta 173.74,58.24,54.19,49.18,31.45 . M S(E S I) ~ m / z$ $162.1\left(\mathrm{M}^{+}+1\right)$; Elemental analysis: calculated: $\mathrm{C}, 52.16, \mathrm{H}$, 9.38, N, 8.69; found: C, 52.10, H, 9.26, N, 8.46.

Compound 3 m (Entry 13, Table 3): Methyl 2-[(benzylamino)<smiles>COC(=O)C(CNc1ccccc1)C(O)c1ccccc1</smiles>

methyl]-3-hydroxy-3-phenylpropanoate, $\mathrm{C}_{18} \mathrm{H}_{21} \mathrm{NO}_{3} 0.270 \mathrm{~g}$ (90\%); white solid; melting point $45^{\circ} \mathrm{C} ;{ }^{1} \mathrm{H}$ NMR $(300 \mathrm{MHz}$, $\left.\mathrm{CDCl}_{3}\right) \delta_{\mathrm{H}}: 2.91(\mathrm{~s}, 2 \mathrm{H}), 3.01(\mathrm{~m}, 2 \mathrm{H}), 3.62(\mathrm{~s}, 3 \mathrm{H}), 3.74(\mathrm{~d}, 2 \mathrm{H})$, 5.12(d, 1H), 7.30(m, Ar H); ${ }^{13} \mathrm{C}$ NMR $(75 \mathrm{MHz}, \mathrm{DMSO}) \delta_{\mathrm{C}}$ : $173.61,141.75,139.39,128.54,128.24,128.03,127.68$, $127.25,126.03,125.73,74.98,53.82,51.76,48.34$; MS(ESI) $\mathrm{m} / \mathrm{z}: 300.01\left(\mathrm{M}^{+}+1\right)$; Elemental analysis: calculated: $C$, 72.22, H, 7.07, N, 4.68; found: C, 72.16, H 7.01, N, 4.62.

Compound 3n (Entry 14, Table 3): Methyl 2-[(benzylamino) methyl]-3-hydroxy-3-(4-nitrophenyl)propanoate,<smiles>COC(=O)C(CNCc1ccccc1)C(O)c1ccc([N+](=O)[O-])cc1</smiles>

$\mathrm{C}_{18} \mathrm{H}_{22} \mathrm{~N}_{2} \mathrm{O}_{5} 0.345 \mathrm{~g}(90 \%)$, sticky compound; ${ }^{1} \mathrm{H}$ NMR $\left(300 \mathrm{MHz}, \mathrm{CDCl}_{3}\right) \delta_{\mathrm{H}}: 3.5(\mathrm{~m}, 3 \mathrm{H}), 3.46(\mathrm{~m}, 5 \mathrm{H}), 5.30(\mathrm{~d}, 1 \mathrm{H})$, 5.68(s, 2H), 7.25-8.19(m, ArH). ${ }^{13} \mathrm{C}$ NMR (75 MHz, DMSO) $\delta_{C}: 171.97,148.99,128.79,73.05,53.02$; (ESI) m/z: 345.14 
$\left(\mathrm{M}^{+}+1\right)$; Elemental analysis: calculated $\mathrm{C}, 62.78, \mathrm{H}, 5.85, \mathrm{~N}$, 8.13; found C, 62.48, H, 5.76, N, 8.10.

\section{Compound 30 (Entry 15, Table 3): 3-(Benzylamino)-1,3-di-}<smiles>O=C(CC(NCc1ccccc1)c1ccccc1)c1ccccc1</smiles>

phenylpropan-1-one, $\mathrm{C}_{22} \mathrm{H}_{21} \mathrm{NO} 0.265 \mathrm{~g}$; yield $84 \%$ white solid; mp $47^{\circ} \mathrm{C} ;{ }^{1} \mathrm{H} \mathrm{NMR}\left(\mathrm{CDCl}_{3}, 300 \mathrm{MHz}\right) \delta_{\mathrm{H}}: 8.41(\mathrm{~s}, 1 \mathrm{H})$, $7.80(\mathrm{~m}, 15 \mathrm{H}), 4.8(\mathrm{~s}, 2 \mathrm{H}), 4.45(\mathrm{t}, J=6.27 \mathrm{~Hz}, 1 \mathrm{H}), 3.9(\mathrm{~m}$, $2 \mathrm{H}) ;{ }^{13} \mathrm{C} \mathrm{NMR}\left(\mathrm{CDCl}_{3}, 75 \mathrm{MHz}\right) \delta_{\mathrm{C}}: 195.53,136.62,136.09$, $134.83,133.90,132.88,130.63,129.00,128.88,128.53$, $128.50,126.65,56.51,55.76,44.24 .772,1173,1197,1454$, 2420, 2952, 3027, 3418; MS (ESI), m/z: $316.13\left(\mathrm{M}^{+}+1\right)$; Elemental analysis: calculated $\mathrm{C}, 83.78, \mathrm{H}, 6.71, \mathrm{~N}, 4.44$; found C, 83.84, H, 6.16, N, 4.78.

Compound 3p (Entry 16, Table 3): 3-Hydroxy-2-(imidazol1-ylmethyl)-3-(4-nitrophenyl)propanoic acid methyl ester,<smiles>COC(=O)C(Cn1ccnc1)C(O)c1ccc([N+](=O)[O-])cc1</smiles>

$\mathrm{C}_{14} \mathrm{H}_{15} \mathrm{~N}_{3} \mathrm{O}_{5} 0.273$ g; yield $89 \%$; white solid; $\mathrm{mp} 168{ }^{\circ} \mathrm{C}$; ${ }^{1} \mathrm{H}$ NMR $\left(\mathrm{CDCl}_{3}, 300 \mathrm{MHz}\right) \delta_{\mathrm{H}}: 8.12(\mathrm{~d}, J=3.6 \mathrm{~Hz}, 2 \mathrm{H}), 8.09$ $(\mathrm{m}, 3 \mathrm{H}), 6.87(\mathrm{~m}, 2 \mathrm{H}), 6.75(\mathrm{~s}, 1 \mathrm{H}) 4.89(\mathrm{~d}, J=7.2 \mathrm{~Hz}, 1 \mathrm{H})$, 4.44-4.12(m,3H), $4.20(\mathrm{~d}, J=3.3 \mathrm{~Hz}, 2 \mathrm{H}), 3.11(\mathrm{~s}, 3 \mathrm{H}) ;{ }^{13} \mathrm{C}$ NMR $\left(\mathrm{CDCl}_{3}, 75 \mathrm{MHz}\right) \delta_{\mathrm{C}}: 175.76,152.79,132.04,131.77$, $128.24,123.96,59.54,56.70,44.99,44.16 .757,856,1080$, $1223,1244,1353,1446,1514,1514,1634,1737,2851$, 2959, 3112, 3426; MS (ESI, m/z) $305.99\left(\mathrm{M}^{+}+1\right)$; Elemental analysis: calculated $\mathrm{C}, 55.08, \mathrm{H}, 4.95, \mathrm{~N}, 13.76$; found $\mathrm{C}$, 55.10, H, 4.92, N, 13.78 .

Acknowledgements One of the authors (AT) acknowledges UGC, New Delhi, for the financial assistance in the form of a fellowship during the tenure of the work. The authors also acknowledge IITG for assistance in providing spectra.

\section{Compliance with ethical standards}

Conflict of interest On behalf of all authors, the corresponding author states that there is no conflict of interest.

\section{References}

1. Sarmah M, Dewan A, Mondal M, Thakur AJ, Bora U (2016) Analysis of water extract of waste papaya bark ash and its implications as in situ base in ligandless recyclable Suzuki-Miyaura coupling reaction. RSC Adv 6:28981

2. Mahanta A, Mandal M, Thakur AJ, Bora U (2016) An improved Suzuki-Miyaura cross-coupling reaction with the aid of in situ generated PdNPs: evidence for enhancing effect with biphasic system. Tetrahedron Lett 57:3091

3. Dewan A, Sarmah M, Thakur AJ, Bharali P, Bora U (2018) Greener biogenic approach for the synthesis of palladium nanoparticles using papaya peel: an eco-friendly catalyst for $C-C$ coupling reaction. ACS Omega 3:5327

4. Boruah PR, Ali AA, Saikia B, Sarma D (2015) A novel green protocol for ligand free Suzuki-Miyaura cross-coupling reactions in WEB at room temperature. Green Chem 17:1442

5. Boruah PR, Ali AA, Saikia B, Chetia M, Sarma D (2015) Pd(OAC) in WERSA: a novel green catalytic system for Suzuki Miyaura cross-coupling reactions at room temperature. Chem Commun 51:11489

6. Sarmah M, Neog AB, Boruah PK, Das MR, Bora U (2019) Effect of substrates on catalytic activity of biogenic palladium nanoparticles in C-C cross-coupling reactions. ACS Omega 4:3329

7. Sarmah M, Mondal M, Bora U (2017) Agro-waste extract based solvents: emergence of novel green solvent for the design of sustainable processes in catalysis and organic chemistry. Chem Sel 2:5180

8. Dewan A, Sarmah M, Thakur AJ, Bora U, Thakur AJ (2017) In situ generation of palladium nanoparticles using agro waste and their use as catalyst for copper, amine and ligandfree Sonogashira reaction. Appl Organomet Chem 31:34646

9. Dewan A, Sarmah M, Thakur AJ, Bora U, Thakur AJ (2016) A green protocol for ligand, copper and base free Sonogashira cross-coupling reaction. Tetrahedron Lett 57:3760

10. Saikia B, Borah $P$, Barua NC (2015) $\mathrm{O}_{2}$ in WEB: a highly efficient catalyst system for Dakin reaction. Green Chem 17:4533

11. Laskar K, Paul S, Bora U (2019) Cellulose as recyclable organocatalyst for ipso-hydroxylation of arylboronic acids. Tetrahedron Lett 60:151044

12. Laskar K, Bhattacharjee P, Gohain M, Deka D, Bora U (2019) Application of bio-based green heterogeneous catalyst for the synthesis of arylidinemalononitriles. Sus Chem Pharm $14: 100181$

13. Surneni N, Barua NC, Saikia B (2016) Application of natural feedstock extract: the Henry reaction. Tetrahedron Lett 57:2814

14. Bagul SD, Rajput JD, Bendre RS (2017) Synthesis of 3-carboxycoumarins at room temperature in water extract of banana peels. Environ Chem. https://doi.org/10.1007/s1031 1-017-0645-Z

15. Konwar M, Ali AA, Sarma A (2016) A green protocol for peptide bond formation in WEB. Tetrahedron Lett 57:2283

16. Deka DC, Basumatary S (2011) High quality biodiesel from yellow oleander (Thevetia peruviana) seed oil. Biomass Bioenergy 35:1797

17. Gohain M, Devi A, Deka D (2017) Musa balbisiana Colla peel as highly effective renewable heterogeneous base catalyst for biodiesel production. Ind Crops Prod 109:8

18. Sarma AK, Kumar P, Aslam M, Chouhan AP (2014) Preparation and characterization of Musa balbisiana Colla underground stem nano-material for biodiesel production under elevated conditions. Catal Lett 144:1344

19. Chouhan AP, Sarma AK (2013) Biodiesel production from Jatropha curcas $\mathrm{L}$. oil using Lemna perpusilla Torrey ash as heterogeneous catalyst. Biomass Bioenergy 55:386

20. Vadery V, Narayanan BN, Ramakrishnan RM, Cherikkallinmel SK, Sugunan S, Narayanan DP, Sasidharan S (2014) Room temperature production of jatropha biodiesel over coconut husk ash. Energy 70:588-594 
21. Chen GY, Shan R, Shi JF, Yan BB (2015) Transesterification of palm oil to biodiesel using rice husk ash-based catalysts. Fuel Process Technol 133:8

22. Betiku E, Etim AO, Pereao O, Ojumu TV (2017) Two-step conversion of neem (Azadirachta indica) seed oil into fatty methyl esters using a heterogeneous biomass-based catalyst: an example of cocoa pod husk. Energy Fuels 31:6182

23. Gohain M, Laskar K, Paul AK, Daimary N, Maharana M, Goswami IK, Hazarika A, Bora U, Deka D (2020) Carica papaya stem: a source of versatile heterogeneous catalyst for biodiesel production and C-C bond formation. Renew Energy 147(1):541-555

24. Gohain M, Laskar K, Phukon H, Bora U, Kalita D, Deka D (2020) Towards sustainable biodiesel and chemical production: multifunctional use of heterogeneous catalyst from littered Tectona grandis leaves. Waste Manag 102:212

25. Sarmah M, Dewan A, Thakur AJ, Bora U (2017) Extraction of base from Eichhornia crassipes and its implication in palladiumcatalyzed Suzuki cross-coupling. Sustain Chem 2:7091

26. Talukdar A, Deka DC (2016) Preparation and characterization of a heterogeneous catalyst from water hyacinth (Eichhornia crassipes): catalytic application in the synthesis of bis(indolyl) methanes and bis(pyrrolyl)methanes under solvent free condition. Curr Catal 5:51

27. Gellman SH, Manifesto A (1998) Foldamers: a manifesto. Acc Chem Res 31:173

28. Bartoli G, Cimarelli C, Marcantoni E, Palmieri G, Petrini M (1994) Chemo- and diastereoselective reduction of beta -enamino esters: a convenient synthesis of both cis- and trans-gamma amino alcohols and beta-amino esters. J Org Chem 59:5328

29. Yadav JS, Reddy BVS, Basak AK, Narsaiah AV (2003) Aza-Michael reactions in ionic liquids. a facile synthesis of $\beta$-amino compounds. Chem Lett 32:988-989

30. Genov M, Dimitrov V, Ivanova V (1997) New $\delta$-amino alcohol for the enantioselective addition of dialkylzincs to aldehydes. Tetrahedron Asymmetry 8:3703

31. Corey EJ, Reichard GA (1989) Enantioselective and practical syntheses of $R$ - and $S$-fluoxetines. Tetrahedron Lett 30:395207

32. Cardillo $G$, Tomasini $C$ (1996) Asymmetric synthesis of $\beta$-amino acids and $\alpha$-substituted $\beta$-amino acids. Chem Soc Rev 25:117
33. Loh TP, Wei LL (1998) Indium trichloride-catalyzed conjugate addition of amines to ethylenic compounds in water. Synlett 9:975

34. Bartoli G, Bosco M, Marcantoni E, Petrini M, Sambri L, Torregiani $E$ (2001) Conjugate addition of amines to $a, \beta$-enones promoted by $\mathrm{CeCl} 3 \cdot 7 \mathrm{H} 2 \mathrm{O}-\mathrm{Nal}$ system supported in silica gel. J Org Chem 2001(66):9052

35. Jenner G (1995) Catalytic high pressure synthesis of hindered $\beta$-amino esters. Tetrahedron Lett 36:233

36. Srivastava N, Banik BK (2003) Bismuth nitrate-catalyzed versatile Michael reactions. J Org Chem 68:2109

37. Wabnitz TC, Spencer JB (2002) Convenient synthesis of Cbzprotected $\beta$-amino ketones by a copper-catalysed conjugate addition reaction. Tetrahedron Lett 4:3891

38. Azizi N, Saidi MR (2004) LiClO4 accelerated Michael addition of amines to $a, \beta$-unsaturated olefins under solvent-free conditions. Tetrahedron 60:383

39. Reboule I, Gil R, Collin J (2005) Aza-Michael reactions catalyzed by samarium diiodide. Tetrahedron Lett 46:7761

40. Xu LW, Li L, Xia CG (2004) Transition-metal-based Lewis acid catalysis of aza-type Michael additions of amines to $a$, $\beta$-unsaturated electrophiles in water. Helv Chim Acta 87:1522

41. Duan Z, Xuan X, Li T, Yang C, Wu Y (2006) Cerium(IV) ammonium nitrate (CAN) catalyzed aza-Michael addition of amines to $a, \beta$-unsaturated electrophiles. Tetrahedron Lett 47:5433

42. Wang Y, Yuan YQ, Guo SR (2009) Silica sulfuric acid promotes aza-Michael addition reactions under solvent-free condition as a heterogeneous and reusable catalyst. Molecules 14:4779

43. Mukherjee C, Misra AK (2007) Aza-Michael addition of amines to activated alkenes catalyzed by silica supported perchloric acid under a solvent-free condition. Lett Org Chem 4:54

44. Talukdar A, Deka DC (2019) Chemical analysis of traditional food additive Dokhora khar derived from Water Hyacinth. Curr Nutri Food Sci. https://doi.org/10.2174/15734013156661902061 41528

Publisher's Note Springer Nature remains neutral with regard to jurisdictional claims in published maps and institutional affiliations. 